\title{
DG CATEGORIES AND EXCEPTIONAL COLLECTIONS
}

\author{
AGNIESZKA BODZENTA
}

(Communicated by Harm Derksen)

\begin{abstract}
A description of how to assign to a full exceptional collection on a variety $X$ a DG category $\mathcal{C}$ such that the bounded derived category of coherent sheaves on $X$ is equivalent to the bounded derived category of $\mathcal{C}$ is given in a 1990 work by Bondal and Kapranov, Framed triangulated categories. In this paper we show that the category $\mathcal{C}$ can be chosen to have finite-dimensional spaces of morphisms. We describe how it behaves under mutations and present an algorithm allowing us to calculate it for full exceptional collections with vanishing $\mathrm{Ext}^{k}$ groups for $k>1$.
\end{abstract}

\section{INTRODUCTION}

1.1. Motivation. Derived categories of coherent sheaves have been an object of vivid interest since their discovery in the 1960's. Full exceptional collections are one of the most important tools used to study them. When such a collection is strong the derived category is equivalent to a derived category of modules over some finitedimensional noncommutative algebra; see [Bon89]. On the other hand, the theorem of Orlov Or192] describes how derived categories change while passing to projective bundles over $X$ and under blow-ups. In particular, if derived categories of coherent sheaves on both $X$ and a smooth $Z \subset X$ have full exceptional collections, then the derived category of $Y=\mathrm{Bl}_{Z} X$ also does. Moreover, the exceptional collection on $Y$ can be explicitly given in terms of exceptional collections on $X$ and $Y$. However, if the collections on $X$ and $Z$ are strong the resulting collection on $Y$ does not have to satisfy this condition. Therefore, it is natural to try to understand what description of $D^{b}(X)$ a full exceptional collection, not necessarily strong, gives.

The answer is given in BK90]. Namely, there exists a DG category $\mathcal{C}_{\sigma}$ such that $D^{b}(\operatorname{Coh}(X))$ is equivalent to a bounded derived category of modules over $\mathcal{C}_{\sigma}$. It follows from the construction that $\mathcal{C}_{\sigma}$ has finitely many objects.

However, as $\mathcal{C}_{\sigma}$ is constructed as a subcategory of the category of complexes of injective sheaves on $X$, we a priori do not know anything about the dimension of the spaces of morphisms between any two objects in it. The possibility that the space of morphisms can be infinitely dimensional prevents us, for example, from considering canonical objects such as $E \otimes \operatorname{Hom}(E, F)$, which are crucial for defining the braid group action on the set of exceptional collections; see [Bon89, [BK90]. The main goal of this paper is to show that for smooth projective varieties, the DG category $\mathcal{C}_{\sigma}$ can be chosen to have finite-dimensional spaces of morphisms.

Received by the editors August 2, 2012 and, in revised form, October 19, 2013.

2010 Mathematics Subject Classification. Primary 14F05, 14J26.

The author was partially supported by MNiSW grant number NN 201420639. 
1.2. Main result. Using the theory of $A_{\infty}$ categories, we prove the following fact.

Theorem 1.1. Let $X$ be a smooth projective variety and let $\sigma=\left\langle\mathcal{E}_{1}, \ldots, \mathcal{E}_{n}\right\rangle$ be a full exceptional collection on $X$. Then there exists an ordered, finite $D G$ category $\widetilde{\mathcal{C}_{\sigma}}$ such that $D^{b}(X)$ is equivalent to $D^{b}\left(\widetilde{\mathcal{C}_{\sigma}}\right)$.

Here, we say that a category $\widetilde{\mathcal{C}}$ is ordered if one can partially order objects of $\widetilde{\mathcal{C}}$ in such a way that there are no nontrivial morphisms from $C_{1}$ to $C_{2}$ if $C_{2} \prec C_{1}$. Furthermore, a category $\widetilde{\mathcal{C}}$ is finite if all spaces of morphisms in $\widetilde{\mathcal{C}}$ are finite dimensional.

Thus, $\widetilde{\mathcal{C}_{\sigma}}$ can be presented as a path algebra of a DG quiver, a quiver with grading and differential on arrows.

Knowing that the DG category $\widetilde{\mathcal{C}_{\sigma}}$ can be chosen to be finite we then describe how it can be calculated in some particular cases. One method uses the, already mentioned, fact that the set of full exceptional collections on $X$ admits an action of the braid group. As it has been stated in the original paper BK90, the action of the braid group can be lifted to associated DG categories. It allows us to describe the category $\widetilde{\mathcal{C}_{\sigma}}$ for any collection $\sigma$ which can be mutated to a strong one.

Another way to calculate the DG category $\widetilde{\mathcal{C}_{\sigma}}$ is to use universal extensions introduced by Hille and Perling in [HP11. This method leads to the following result.

Theorem 1.2. Let $\sigma=\left\langle\mathcal{E}_{1}, \ldots, \mathcal{E}_{n}\right\rangle$ be a full exceptional collection on a smooth projective variety $X$ such that $\operatorname{Ext}^{k}\left(\mathcal{E}_{i}, \mathcal{E}_{j}\right)=0$ for $k \neq 0,1$ and any $i, j$. Then the $D G$ category $\widetilde{\mathcal{C}_{\sigma}}$ can be calculated by means of universal extensions.

From the above theorem and the discussion preceding we conclude the following fact.

Corollary 1.3. Let $X$ be a smooth projective variety and let $\sigma=\left\langle\mathcal{E}_{1}, \ldots, \mathcal{E}_{n}\right\rangle$ be a full exceptional collection on $X$ which either can be mutated to a strong exceptional collection or satisfies the condition $\operatorname{Ext}^{k}\left(\mathcal{E}_{i}, \mathcal{E}_{j}\right)=0$ for $k>1$ and all $i, j$. Then the bounded derived category of coherent sheaves on $X$ is equivalent to the homotopy category of modules over a finite and ordered $D G$ category $\mathcal{C}_{\sigma}$. The $D G$ category $\mathcal{C}_{\sigma}$ can be presented as a DG category associated to a finite $D G$ quiver which can be explicitly calculated.

In particular, the assumptions of the above corollary are satisfied by full exceptional collections of line bundles on smooth rational surfaces constructed by Hille and Perling in [HP11. As an example we present a DG quiver of a full exceptional collection on $\mathbb{P}^{2}$ blown up along a subscheme of degree 2 supported at one point.

Full strong exceptional collections have also been used in AKO06, HI13. and Per10 to describe noncommutative deformations of varieties. Knowledge of the DG categories of exceptional collections, which are not strong, can lead to a generalisation of this idea. As an example we present a noncommutative deformation from $\mathbb{P}^{2}$ blown up in two different points to $\mathbb{P}^{2}$ blown up along a subscheme of degree 2 supported at one point.

\section{ENHANCED TRIANGUlated CATEGORIES}

Let us recall definitions and facts about enhanced triangulated categories and exceptional collections after [Bon89, BK90, BLL04] and Kel94]. 
2.1. Exceptional collections and mutations. Let $\mathcal{D}$ be a $\mathbb{C}$-linear triangulated category. An object $E \in \mathcal{D}$ is called exceptional if $\mathcal{D}(E, E)=\mathbb{C}$ and $\mathcal{D}(E, E[i])=0$ for $i \neq 0$. An ordered collection $\sigma=\left\langle E_{1}, \ldots, E_{n}\right\rangle$ of exceptional objects is called an exceptional collection if $\mathcal{D}\left(E_{i}, E_{j}[k]\right)=0$ for $i>j$ and all $k$. An exceptional collection $\sigma$ is strong if we also have $\mathcal{D}\left(E_{i}, E_{j}[k]\right)=0$ for $k \neq 0$ and all $i, j$. It is full if the smallest strictly full subcategory of $\mathcal{D}$ containing $E_{1}, \ldots, E_{n}$ is equal to $\mathcal{D}$.

Let $\langle E, F\rangle$ be an exceptional pair in $\mathcal{D}$. Then $\left\langle L_{E} F, E\right\rangle$ and $\left\langle F, R_{F} E\right\rangle$ are also exceptional pairs for $L_{E} F$ and $R_{F} E$ defined in Bon89] by means of distinguished triangles in $\mathcal{D}$ :

$$
\begin{aligned}
L_{E} F & \rightarrow \mathcal{H o m}_{\mathcal{D}}(E, F) \otimes E \\
E & \rightarrow F \operatorname{Hom}_{\mathcal{D}}(E, F)^{*} \otimes F \rightarrow L_{E} F[1], \\
& \otimes \rightarrow E[1] .
\end{aligned}
$$

Here, $\mathcal{H o m}_{\mathcal{D}}(E, F)$ denotes a complex of $\mathbb{C}$-vector spaces with trivial differential; $\mathcal{H o m}_{\mathcal{D}}(E, F)=\bigoplus \mathcal{D}(E, F[k])$. For an element $E \in \mathcal{D}$ and a complex $V^{\bullet}$ the tensor product is defined by $E \otimes V^{\bullet}=\bigoplus_{k \in \mathbb{Z}} \bigoplus_{i=0}^{\operatorname{dim} V^{k}} E[-k]$.

For an exceptional collection $\sigma=\left\langle E_{1}, \ldots, E_{n}\right\rangle$ the $i$-th left mutation $L_{i} \sigma$ and the $i$-th right mutation $R_{i} \sigma$ are defined by

$$
\begin{array}{r}
L_{i} \sigma=\left\langle E_{1}, \ldots, E_{i-1}, L_{E_{i}} E_{i+1}, E_{i}, E_{i+2}, \ldots, E_{n}\right\rangle, \\
R_{i} \sigma=\left\langle E_{1}, \ldots, E_{i-1}, E_{i+1}, R_{E_{i+1}} E_{i}, E_{i+2}, \ldots, E_{n}\right\rangle .
\end{array}
$$

Mutations define an action of the braid group on the set of full exceptional collections.

\subsection{Enhanced triangulated categories.}

Definition 2.1. A $D G$ category is a preadditive category $\mathcal{C}$ in which abelian groups $\mathcal{C}(A, B)$ are endowed with a $\mathbb{Z}$-grading and a differential $\partial$ of degree one. The composition of morphisms

$$
\mathcal{C}(B, C) \otimes \mathcal{C}(A, B) \rightarrow \mathcal{C}(A, C)
$$

is a morphism of complexes and for any object $C \in \mathcal{C}$ the identity morphism $\operatorname{id}_{C}$ is a closed morphism of degree zero.

For an element $x$ in a graded vector space we will denote by $|x|$ the grading of $x$. By $\mathcal{C}^{i}(A, B)$ we will denote morphisms of degree $i$.

A DG category $\mathcal{C}$ is ordered if there exists a partial order $\preceq$ on the set of objects such that $\mathcal{C}(A, B)=0$ for $B \prec A$. It is finite if the set of objects of $\mathcal{C}$ is finite and for any $C_{1}, C_{2} \in \mathcal{C}$ the vector space $\mathcal{C}\left(C_{1}, C_{2}\right)$ is finite dimensional.

To a DG category $\mathcal{C}$ we associate three categories. The graded category $\mathcal{C}^{\text {gr }}$ is obtained from $\mathcal{C}$ by forgetting the differentials on morphisms while the homotopy category $H(\mathcal{C})$ has the same objects as $\mathcal{C}$ and morphisms given by the cohomology groups of morphisms in $\mathcal{C}$. A further restriction to the zeroth cohomology gives a preadditive category $H^{0}(\mathcal{C})$.

A morphism $s: C \rightarrow C^{\prime}$ in $\mathcal{C}$ is a homotopy equivalence if $H(s)$ is an isomorphism. Then we say that $C$ and $C^{\prime}$ are homotopy equivalent.

A $D G$ functor between two DG categories $\mathcal{C}$ and $\mathcal{C}^{\prime}$ is an additive functor $F: \mathcal{C} \rightarrow \mathcal{C}^{\prime}$ which preserves the grading and differential on morphisms. DG functors between DG categories form a DG category. 
Let $F, G: \mathcal{C}_{1} \rightarrow \mathcal{C}_{2}$ be DG functors. To construct a DG category DG-Fun $\left(\mathcal{C}_{1}, \mathcal{C}_{2}\right)$ we put $\operatorname{DG}-\mathrm{Fun}\left(\mathcal{C}_{1}, \mathcal{C}_{2}\right)^{k}(F, G)$ to be the set of natural transformations $t: F^{\mathrm{gr}} \rightarrow G^{\mathrm{gr}}[k]$ (i.e. for $C \in \mathcal{C}_{1}$ we have $\left.t_{C} \in \mathcal{C}_{2}^{k}(F(C), G(C))\right)$. The differential $\partial$ is defined pointwise; for $t_{C}: F(C) \rightarrow G[k](C)$ we have $(\partial(t))_{C}=\partial\left(t_{C}\right): F(C) \rightarrow G[k+1](C)$.

The category of contravariant DG functors is denoted by DG-Fun ${ }^{\circ}\left(\mathcal{C}, \mathcal{C}^{\prime}\right)$. A functor $F: \mathcal{C} \rightarrow \mathcal{C}^{\prime}$ is a quasi-equivalence if $H(F): H(\mathcal{C}) \rightarrow H\left(\mathcal{C}^{\prime}\right)$ is an equivalence.

The category DGVect $\mathbb{C}$ of complexes of vector spaces with homogeneous morphisms $f: V^{\bullet} \rightarrow W^{\bullet}, f\left(V^{i}\right) \subset W^{i+k}$ and a differential

$$
\partial(f)^{i}=\partial_{W} f^{i+1}-(-1)^{|f|} f^{i+1} \partial_{V}
$$

is a DG category.

A right $D G$ module $M$ over a DG category $C$ is an element of $\operatorname{DG}$ Fun $^{\circ}\left(\mathcal{C}\right.$, DGVect $\left._{\mathbb{C}}\right)$. After Kel94 we define a derived category $D(\mathcal{C})$ as a localization of $H^{0}\left(\mathrm{DG}_{\mathrm{C}} \mathrm{Fun}^{\mathrm{O}}\left(\mathcal{C}, \mathrm{DGVec}_{\mathbb{C}}\right)\right)$ with respect to the class of homotopy equivalences. By $D^{b}(\mathcal{C})$ we will denote the subcategory of $D(\mathcal{C})$ formed by compact objects. The Yoneda embedding gives a functor $h: \mathcal{C} \rightarrow D^{b}(\mathcal{C})$ which assigns to every $C \in \mathcal{C}$ a free module $h_{C}=\mathcal{C}(-, C)$.

For a DG category $\mathcal{C}$ we define the category $\widehat{\mathcal{C}}$ of formal shifts. The objects of $\widehat{\mathcal{C}}$ are $C[n]$ where $C \in \mathcal{C}$ and $n \in \mathbb{N}$. For elements $C_{1}[k]$ and $C_{2}[n]$ of $\widehat{\mathcal{C}}$ we put $\operatorname{Hom}_{\widehat{\mathcal{C}}}^{l}\left(C_{1}[k], C_{2}[n]\right)=\operatorname{Hom}_{\mathcal{C}}^{l+n-k}\left(C_{1}, C_{2}\right)$. For appropriate sign convention see BLL04.

Let $B, C$ be objects of a DG category $\mathcal{C}$ and let $f \in \mathcal{C}(B, C)$ be a closed morphism. Assume that $B[1]$ is also an object of $\mathcal{C}$; i.e. there exists an object $B^{\prime}$ and closed morphisms $t: B \rightarrow B^{\prime}, t^{\prime}: B^{\prime} \rightarrow B$ of degree 1 and -1 respectively such that $t^{\prime} t=\mathrm{id}_{B}$ and $t t^{\prime}=\mathrm{id}_{B^{\prime}}$. An object $D \in \mathcal{C}$ is called a cone of $f$ if there exist morphisms

$$
B^{\prime} \stackrel{i}{\longrightarrow} D \stackrel{p}{\longrightarrow} B^{\prime}
$$

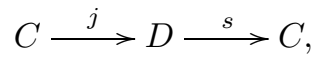

such that

$$
\begin{aligned}
& p i=1, \quad s j=1, \quad s i=0, \quad p j=0, \quad i p+j s=1, \\
& \partial(j)=0, \quad \partial(p)=0, \quad \partial(i)=j f, \quad \partial(s)=-f p .
\end{aligned}
$$

It is proved in BLL04 that the cone of closed degree zero morphism is uniquely defined up to a DG isomorphism.

One can formally add cones of closed morphisms to a DG category $\mathcal{C}$ by considering the category $\mathcal{C}^{\text {pre-tr }}$ of one-sided twisted complexes over $\mathcal{C}$.

Definition 2.2. A one-sided twisted complex over a DG category $\mathcal{C}$ is an expression $\left(\bigoplus_{i=1}^{n} C_{i}\left[r_{i}\right], q_{i, j}\right)$ where the $C_{i}$ 's are objects of $\mathcal{C}, r_{i} \in \mathbb{Z}, n \geq 0$ and $q_{i, j} \in$ $\mathcal{C}^{1}\left(C_{i}\left[r_{i}\right], C_{j}\left[r_{j}\right]\right)$ such that $q_{i, j}=0$ for $i \geq j$ and $\partial q+q^{2}=0$.

One-sided twisted complexes over $\mathcal{C}$ form a DG category $\mathcal{C}^{\text {pre-tr }}$ with the morphism space between $C=\left(\bigoplus C_{i}\left[r_{i}\right], q\right)$ and $C^{\prime}=\left(\bigoplus C_{j}^{\prime}\left[r_{j}^{\prime}\right], q^{\prime}\right)$ given by the set of matrices $f=\left(f_{i, j}\right), f_{i, j}: C_{i}\left[r_{i}\right] \rightarrow C_{j}^{\prime}\left[r_{j}^{\prime}\right]$. With a differential defined as

$$
\partial(f)=\left(\partial f_{i, j}\right)+q^{\prime} f-(-1)^{\operatorname{deg} f} f q
$$

the category $\mathcal{C}^{\text {pre-tr }}$ is a DG category. Its zeroth homotopy category is $\mathcal{C}^{\text {tr }}$. 
Let $C=\left(\bigoplus C_{i}\left[r_{i}\right], q_{i, j}\right)$ be an object of $\mathcal{C}^{\text {pre-tr }}$. The shift of $C$ is defined as $C[1]=\left(\bigoplus C_{i}\left[r_{i}+1\right],-q_{i, j}\right)$. Clearly, the category $\mathcal{C}^{\text {pre-tr }}$ is closed under shifts.

Let $f: C \rightarrow D$ be a closed morphism of degree zero in $\mathcal{C}^{\text {pre-tr }}$, where $C=\left(\bigoplus_{i=1}^{n} C_{i}\left[r_{i}\right], q_{i, j}\right), D=\left(\bigoplus_{i=1}^{m} D_{i}\left[s_{i}\right], p_{i, j}\right)$. The cone of $f$ is a twisted complex Cone $(f)=\left(\bigoplus_{i=1}^{n+m} E_{i}\left[t_{i}\right], u_{i, j}\right)$, where

$$
\begin{aligned}
& E_{i}= \begin{cases}C_{i} & \text { for } i \leq n, \\
D_{i-n} & \text { for } i>n,\end{cases} \\
& t_{i}=\left\{\begin{array}{ll}
r_{i}+1 & \text { for } i \leq n, \\
s_{i} & \text { for } i>n,
\end{array} \quad u_{i, j}= \begin{cases}q_{i, j} & \text { for } i, j \leq n, \\
f_{i, j-n} & \text { for } i \leq n<j, \\
p_{i-n, j-n} & \text { for } i, j>n .\end{cases} \right.
\end{aligned}
$$

The convolution functor Tot $:\left(\mathcal{C}^{\text {pre-tr }}\right)^{\text {pre-tr }} \rightarrow \mathcal{C}^{\text {pre-tr }}$ establishes a quasiequivalence between $\left(\mathcal{C}^{\text {pre-tr }}\right)^{\text {pre-tr }}$ and $\mathcal{C}^{\text {pre-tr }}$. Let $C_{i}=\left(\bigoplus_{j=1}^{n_{i}} D_{j}^{i}\left[r_{j}^{i}\right], q_{j k}^{i}\right)$ be objects of $\mathcal{C}^{\text {pre-tr }}$ and let $C=\left(\bigoplus_{i=1}^{n} C_{i}\left[r_{i}\right], q_{i j}\right)$ be a twisted complex in $\left(\mathcal{C}^{\text {pre-tr }}\right)^{\text {pre-tr }}$. Then the convolution $\operatorname{Tot}(C)$ is equal to $\left(\bigoplus_{i=1}^{n} \bigoplus_{j=1}^{n_{j}^{i}} D_{j}^{i}\left[r_{j}^{i}+r_{i}\right], q_{j k}^{i}+q_{i j}\right)$.

The DG category $\mathcal{C}$ is pretriangulated if the embedding $H^{0}(\mathcal{C}) \rightarrow \mathcal{C}^{t r}$ is an equivalence. The category $H^{0}(\mathcal{C})$ for a pretriangulated category is triangulated.

A triangulated category $\mathcal{D}$ is enhanced if it has an enhancement - a pretriangulated category $\mathcal{C}$ such that $\mathcal{D}$ is equivalent to $H^{0}(\mathcal{C})$.

The category $\mathcal{C}^{\text {tr }}$ need not to be Karoubian. As shown in BLL04 the category $D^{b}(\mathcal{C})$ is the Karoubization of $\mathcal{C}^{\text {tr }}$.

A standard example of an enhanced triangulated category is the derived category $D(\mathcal{A})$ of an abelian category $\mathcal{A}$ with enough injectives $I$. Its enhancement is the category of complexes of injective sheaves $\operatorname{Kom}(I)$.

With the above definitions we are ready to state the following theorem.

Theorem 2.3 (Theorem 1 of [BK90]). Let $\widetilde{\mathcal{C}}$ be a pretriangulated category, $E_{1}, \ldots, E_{n}$ objects of $\widetilde{\mathcal{C}}$ and $\mathcal{C} \subset \widetilde{\mathcal{C}}$ the full $D G$ subcategory on the objects $E_{i}$. Then the smallest triangulated subcategory of $H^{0}(\widetilde{\mathcal{C}})$ containing $E_{1}, \ldots, E_{n}$ is equivalent to $\mathcal{C}^{\text {tr }}$ as a triangulated category.

It follows that a full exceptional collection $\sigma=\left\langle E_{1}, \ldots, E_{n}\right\rangle$ in an enhanced triangulated category $\mathcal{D}$ leads to an equivalence of $\mathcal{D}$ and $\mathcal{C}_{\sigma}^{\text {tr }}$ for some DG category $\mathcal{C}_{\sigma}$. The category $\mathcal{C}_{\sigma}$ is a subcategory of the enhancement of $\mathcal{D}$ and thus $H^{i}\left(\mathcal{C}_{\sigma}(E, F)\right)=\mathcal{D}(E, F[i])$ for any elements $E, F$ of $\sigma$.

Remark 2.4 (see BLL04]). If an enhanced triangulated category $\mathcal{D}$ is Karoubian, then a full exceptional collection $\sigma$ leads to an equivalence of $\mathcal{D}$ and $D^{b}\left(\mathcal{C}_{\sigma}\right)$.

\section{Properties of DG CATEgories associated TO FULL EXCEPTIONAL COLLECTIONS}

From now on let $X$ be a smooth projective variety. Let $D^{b}(X)=D^{b}(\operatorname{Coh}(X))$ denote the bounded derived category of the category of coherent sheaves on $X$. It is an enhanced Karoubian triangulated category. Its enhancement is, for example, given by the DG category $\mathrm{Kom}^{b}(I)$ of complexes of injective sheaves bounded from below and with finitely many nonzero coherent cohomology sheaves.

Let $\sigma=\left\langle\mathcal{E}_{1}, \ldots, \mathcal{E}_{n}\right\rangle$ be a full exceptional collection of coherent sheaves on $X$. By Theorem 2.3 there exists a DG category $\mathcal{C}_{\sigma}$ with objects $\mathcal{E}_{1}, \ldots, \mathcal{E}_{n}$ such that $D^{b}(X)$ is equivalent to $\mathcal{C}_{\sigma}^{\text {tr }}$. The category $D^{b}(X)$ is Karoubian so $D^{b}(X)$ and $D^{b}\left(\mathcal{C}_{\sigma}\right)$ 
are equivalent. As $\sigma$ is an exceptional collection the category $H\left(\mathcal{C}_{\sigma}\right)$ is ordered and finite.

Remark 3.1. If a DG category $\mathcal{C}$ has only zeroth cohomology, then it is quasiequivalent to $H(\mathcal{C})=H^{0}(\mathcal{C})$. Indeed, for $C_{1}, C_{2} \in \mathcal{C}$ let $\mathcal{C}\left(C_{1}, C_{2}\right)=$ $\bigoplus_{n \in \mathbb{Z}} \mathcal{C}^{n}\left(C_{1}, C_{2}\right)$ with differential

$$
\partial_{C_{1}, C_{2}}^{n}: \mathcal{C}^{n}\left(C_{1}, C_{2}\right) \rightarrow \mathcal{C}^{n+1}\left(C_{1}, C_{2}\right)
$$

and let $\mathcal{C}_{I}$ be a DG category such that ob $\mathcal{C}_{I}=$ ob $\mathcal{C}$ and

$$
\mathcal{C}_{I}\left(C_{1}, C_{2}\right)=\bigoplus_{n<0} \mathcal{C}^{n}\left(C_{1}, C_{2}\right) \oplus \operatorname{ker} \partial_{C_{1}, C_{2}}^{0}
$$

Then the natural inclusion functor $\mathcal{C}_{I} \rightarrow \mathcal{C}$ is a quasi-equivalence. Let us also set

$$
J_{C_{1}, C_{2}}=\operatorname{Im}\left(\partial_{C_{1}, C_{2}}^{-1}\right) \oplus\left(\bigoplus_{n<0} \mathcal{C}^{n}\left(C_{1}, C_{2}\right)\right)
$$

for any $C_{1}$ and $C_{2}$ in $\mathcal{C}$ and consider the category $\mathcal{C}_{I / J}$ with ob $\mathcal{C}_{I / J}=$ ob $\mathcal{C}$ and

$$
\mathcal{C}_{I / J}\left(C_{1}, C_{2}\right)=\mathcal{C}_{I}\left(C_{1}, C_{2}\right) / J_{C_{1}, C_{2}} .
$$

Then $\mathcal{C}_{I / J}$ is isomorphic to $H(\mathcal{C})$ and the natural functor $\mathcal{C}_{I} \rightarrow \mathcal{C}_{I / J}$ is a quasiequivalence.

It shows that if $\sigma$ is a strong exceptional collection, then the DG category $\mathcal{C}_{\sigma}$ is quasi-equivalent to an ordinary category.

3.1. Finiteness. Let $D=D^{b}(\operatorname{Coh}(X))$ and let us consider its enhancement given by the category $\underline{\operatorname{Kom}}^{b}(I)$ of complexes of injective sheaves with finitely many nonzero coherent cohomology. Then, calculating the category $\mathcal{C}_{\sigma}$ requires taking injective resolutions. This suggests that the category $\mathcal{C}_{\sigma}$ can have infinitelydimensional spaces of morphisms. However, it is $A_{\infty}$-quasi-equivalent to an ordered DG category with finite-dimensional morphisms between objects. To prove this let us first recall definitions and properties of $A_{\infty}$ categories after [Kel06] and [LH03].

\subsection{1. $A_{\infty}$ categories.}

Definition 3.2. An $A_{\infty}$ category $\mathcal{A}$ over $\mathbb{C}$ consists of

- objects $\operatorname{ob}(\mathcal{A})$,

- for any two $A, B \in \mathrm{ob}(\mathcal{A})$ a $\mathbb{Z}$-graded $\mathbb{C}$-vector space $\mathcal{A}(A, B)$,

- for any $n \geq 1$ and a sequence $A_{0}, A_{1}, \ldots, A_{n} \in \mathrm{ob}(\mathcal{A})$ a graded map:

$$
m_{n}: \mathcal{A}\left(A_{n-1}, A_{n}\right) \otimes \ldots \otimes \mathcal{A}\left(A_{0}, A_{1}\right) \rightarrow \mathcal{A}\left(A_{0}, A_{n}\right)
$$

of degree $2-n$ such that for any $n$

$$
\sum_{r+s+t=n}(-1)^{r+s t} m_{r+1+t}\left(\mathrm{id}^{\otimes r} \otimes m_{s} \otimes \mathrm{id}^{\otimes t}\right)=0 .
$$

When these formulae are applied to elements additional signs appear because of the Koszul sign rule:

$$
(f \otimes g)(x \otimes y)=(-1)^{|x||g|} f(x) \otimes g(y) .
$$

An $A_{\infty}$ algebra is an $A_{\infty}$ category with one object.

An $A_{\infty}$ category $\mathcal{A}$ is ordered if there exists a partial order $\preceq$ on the $\operatorname{set} \operatorname{ob}(\mathcal{A})$ such that $\mathcal{A}\left(A, A^{\prime}\right)=0$ for $A^{\prime} \prec A$. It is finite if $\mathrm{ob}(\mathcal{A})$ is a finite set and $\mathcal{A}\left(A, A^{\prime}\right)$ is finite dimensional for any $A$ and $A^{\prime}$. 
The operation $m_{1}$ gives for any $A, B \in \mathrm{ob}(\mathcal{A})$ a structure of a complex on $\mathcal{A}(A, B)$. Using $m_{1}$, analogously as for DG categories, we can associate a graded category $H(\mathcal{A})$ to an $A_{\infty}$ category.

The $A_{\infty}$ category is minimal if the operation $m_{1}$ is trivial.

Any DG category is an $A_{\infty}$ category with trivial $m_{i}$ 's for $i>2$.

For any set $S$ there exists an $A_{\infty}$ category $\mathbb{C}\{S\}$. The objects of $\mathbb{C}\{S\}$ are elements of $S$ and

$$
\mathbb{C}\{S\}\left(s_{1}, s_{2}\right)= \begin{cases}\mathbb{C} & \text { if } s_{1}=s_{2}, \\ 0 & \text { otherwise. }\end{cases}
$$

All operations $m_{n}$ in $\mathbb{C}\{S\}$ are trivial.

Definition 3.3. A functor of $A_{\infty}$ categories $F: \mathcal{A} \rightarrow \mathcal{B}$ is a map $F_{0}: \mathrm{ob}(\mathcal{A}) \rightarrow \mathrm{ob}(\mathcal{B})$ and a family of graded maps

$$
F_{n}: \mathcal{A}\left(A_{n-1}, A_{n}\right) \otimes \ldots \otimes \mathcal{A}\left(A_{0}, A_{1}\right) \rightarrow \mathcal{B}\left(F_{0}\left(A_{0}\right), F_{0}\left(A_{n}\right)\right)
$$

of degree $1-n$ such that

$$
\sum_{r+s+t=n}(-1)^{r+s t} F_{r+1+t}\left(\mathrm{id}^{\otimes r} \otimes m_{s} \otimes \mathrm{id}^{\otimes t}\right)=\sum_{i_{1}+\ldots+i_{r}=n}(-1)^{p} m_{r}\left(F_{i_{1}} \otimes \ldots \otimes F_{i_{r}}\right),
$$

where $p=(r-1)\left(i_{1}-1\right)+(r-2)\left(i_{2}-1\right)+\ldots+2\left(i_{r-2}-1\right)+\left(i_{r-1}-1\right)$.

Composition of functors is given by

$$
(F \circ G)_{n}=\sum_{i_{1}+\ldots+i_{s}=n} F_{s} \circ\left(G_{i_{1}} \otimes \ldots \otimes G_{i_{s}}\right) .
$$

A functor $F: \mathcal{A} \rightarrow \mathcal{B}$ is called an $A_{\infty}$-quasi-equivalence if $F_{1}$ induces an equivalence between $H(\mathcal{A})$ and $H(\mathcal{B})$.

An $A_{\infty}$ category $\mathcal{A}$ is strictly unital if for any object $A \in \operatorname{ob}(\mathcal{A})$ there exists a morphism $1_{A} \in \mathcal{A}(A, A)$ of degree 0 such that for any $A^{\prime}$ in $\mathcal{A}$ and any morphisms $\phi \in \mathcal{A}\left(A, A^{\prime}\right), \psi \in \mathcal{A}\left(A^{\prime}, A\right)$ we have $m_{2}\left(\phi, 1_{A}\right)=\phi$ and $m_{2}\left(1_{A}, \psi\right)=\psi$. Moreover, for $n \neq 2$ the operation $m_{n}$ equals 0 if any of its arguments is equal to $1_{A}$.

In particular, for any set $S$ the category $\mathbb{C}\{S\}$ is strictly unital.

The category $\mathcal{A}$ is homologically unital if there exist units for the homotopy category $H(\mathcal{A})$.

Proposition 3.4 (Theorem 3.2.1.1 of [LH03]). A minimal homologically unital $A_{\infty}$ category is $A_{\infty}$-quasi-equivalent to a minimal strictly unital $A_{\infty}$ category.

Remark 3.5. A minimal $A_{\infty}$ category is equal to its homotopy category. Hence, an $A_{\infty}$-quasi-isomorphism $F$ given by the above proposition satisfies $F_{1}=$ id.

An $A_{\infty}$ category $\mathcal{A}$ is augmented if there exists a strict unit preserving functor $\epsilon: \mathbb{C}\{\mathrm{ob}(\mathcal{A})\} \rightarrow \mathcal{A}$. Then $\mathcal{A}$ decomposes as $\mathcal{A}=\mathbb{C}\{\mathrm{ob}(\mathcal{A})\} \oplus \overline{\mathcal{A}}$.

3.1.2. Minimal model. Any $A_{\infty}$ category $\mathcal{A}$ is $A_{\infty}$-quasi-equivalent to its homotopy category $H(\mathcal{A})$.

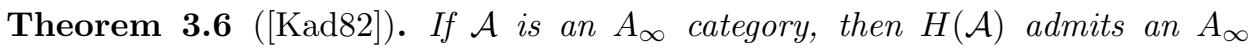
category structure such that

(1) $m_{1}=0$ and $m_{2}$ is induced from $m_{2}^{A}$ and

(2) there is an $A_{\infty}$-quasi-equivalence $\mathcal{A} \rightarrow H(\mathcal{A})$.

Moreover, this structure is unique up to a nonunique $A_{\infty}$-equivalence.

The $A_{\infty}$ category $H(\mathcal{A})$ is called the minimal model of $\mathcal{A}$. 
Remark 3.7. Let $\mathcal{C}$ be a DG category. Its minimal model $H(\mathcal{C})$ is $A_{\infty}$-quasiequivalent to a strictly unital $A_{\infty}$ category. Indeed, the category $\mathcal{C}$ is strictly unital and hence homologically unital. Its homotopy category is also homologically unital and Proposition 3.4 guarantees that there exists a strictly unital minimal category $A_{\infty}$-quasi-equivalent to $H(\mathcal{C})$.

3.1.3. The universal $D G$ category. For any augmented $A_{\infty}$ category $\mathcal{A}$ there exists a DG category $U(\mathcal{A})$ and an $A_{\infty}$-quasi-isomorphism $\mathcal{A} \rightarrow U(\mathcal{A})$. To define the category $U(\mathcal{A})$ we need the following definitions (see further [LH03]).

Definition 3.8. A $D G$ cocategory $\mathcal{B}$ consists of

- the set of objects $B_{i} \in \mathrm{ob}(\mathcal{B})$,

- for any pair of objects $B_{i}, B_{j} \in \mathrm{ob}(\mathcal{B})$ a complex of $\mathbb{C}$-vector spaces $\mathcal{B}\left(B_{i}, B_{j}\right)$ with a differential $d^{i j}$ of degree 1 and

- a coassociative cocomposition - a family of linear maps

$$
\Delta: \mathcal{B}\left(B_{i}, B_{j}\right) \rightarrow \sum_{B_{k} \in \operatorname{ob}(\mathcal{B})} \mathcal{B}\left(B_{k}, B_{j}\right) \otimes \mathcal{B}\left(B_{i}, B_{j}\right)
$$

These data have to satisfy the condition

$$
\Delta \circ d=(d \otimes \mathrm{id}+\mathrm{id} \otimes d) \circ \Delta .
$$

For any set $S$ the $A_{\infty}$ category $\mathbb{C}\{S\}$ is also a DG cocategory.

A functor $\Phi$ between DG cocategories $\mathcal{B}$ and $\mathcal{B}^{\prime}$ preserves the grading and differentials on morphisms and satisfies the condition

$$
\Delta \circ \Phi=(\Phi \otimes \Phi) \circ \Delta .
$$

A DG cocategory $\mathcal{B}$ is counital if it admits a counit - a functor $\eta: \mathcal{B} \rightarrow \mathbb{C}\{\mathrm{ob}(\mathcal{B})\}$. The category $\mathcal{B}$ is coaugmented if it is counital and admits a coaugmentation functor $\varepsilon: \mathbb{C}\{\operatorname{ob}(\mathcal{B})\} \rightarrow \mathcal{B}$ such that the composition $\eta \varepsilon$ is the identity on $\mathbb{C}\{\mathrm{ob}(\mathcal{B})\}$.

Let $\mathcal{B}$ be a coaugmented DG cocategory. Denote by $\overline{\mathcal{B}}$ a cocategory with the same objects as $\mathcal{B}$ and morphisms $\overline{\mathcal{B}}\left(B_{i}, B_{j}\right)=\operatorname{ker} \varepsilon$.

For an augmented $A_{\infty}$ category $\mathcal{A}$ one can define its bar DG cocategory $B_{\infty}(\mathcal{A})$. Recall that as an augmented category $\mathcal{A}$ can be written as $\overline{\mathcal{A}} \oplus \mathbb{C}\{\mathrm{ob}(\mathcal{A})\}$. Then $B_{\infty}(\mathcal{A})=T^{c}(S \overline{\mathcal{A}})$ is a tensor cocategory of the suspension of $\overline{\mathcal{A}}$. Here $S \overline{\mathcal{A}}$ denotes the category $\overline{\mathcal{A}}$ with a shift in the spaces of morphisms $(S \overline{\mathcal{A}})^{n}\left(A, A^{\prime}\right)=\overline{\mathcal{A}}^{n+1}\left(A, A^{\prime}\right)$. $S \overline{\mathcal{A}}$ is not an $A_{\infty}$ category, however the operations $m_{n}$ in $\overline{\mathcal{A}}$ define graded maps of degree 1 in $S \overline{\mathcal{A}}$ :

$$
\begin{gathered}
b_{n}: S \overline{\mathcal{A}}\left(A_{n-1}, A_{n}\right) \otimes \ldots \otimes S \overline{\mathcal{A}}\left(A_{0}, A_{1}\right) \rightarrow S \overline{\mathcal{A}}\left(A_{0}, A_{n}\right), \\
b_{n}=-s \circ m_{n} \circ \omega^{\otimes n},
\end{gathered}
$$

where $s: V \rightarrow S V$ is a suspension of a graded vector space $V$ and $\omega=s^{-1}$.

The cocategory $B_{\infty}(\mathcal{A})$ has the same objects as $\mathcal{A}$ and

$$
\begin{aligned}
B_{\infty}(\mathcal{A})\left(A, A^{\prime}\right)=S \overline{\mathcal{A}}\left(A, A^{\prime}\right) \oplus \bigoplus_{A_{1} \in \mathrm{ob}(\mathcal{A})} S \overline{\mathcal{A}}\left(A_{1}, A^{\prime}\right) \otimes S \overline{\mathcal{A}}\left(A, A_{1}\right) \\
\oplus \bigoplus_{A_{1}, A_{2} \in \mathrm{ob}(\mathcal{A})} S \overline{\mathcal{A}}\left(A_{2}, A^{\prime}\right) \otimes S \overline{\mathcal{A}}\left(A_{1}, A_{2}\right) \otimes S \overline{\mathcal{A}}\left(A, A_{1}\right) \oplus \ldots
\end{aligned}
$$


for $A \neq A^{\prime}$. In the case $A=A^{\prime}$ we have to add $1_{A}$ in degree 0 to the above sum. To simplify the notation we shall write $\left(\alpha_{n}, \ldots, \alpha_{1}\right)$ for $\alpha_{n} \otimes \ldots \otimes \alpha_{1}$. The differential in $B_{\infty}(\mathcal{A})$ is given by

$$
\begin{aligned}
& d\left(\alpha_{n}, \ldots, \alpha_{1}\right) \\
& =\sum_{k=1}^{n} \sum_{l=1}^{n-k+1}(-1)^{\left|\alpha_{l-1}\right|+\ldots+\left|\alpha_{1}\right|}\left(\alpha_{n}, \ldots \alpha_{l+k}, b_{k}\left(\alpha_{l+k-1}, \ldots, \alpha_{l}\right), \alpha_{l-1}, \ldots \alpha_{1}\right),
\end{aligned}
$$

for $\left(\alpha_{n}, \ldots, \alpha_{1}\right) \in B_{\infty}(\mathcal{A})\left(A, A^{\prime}\right)$. The cocomposition is given by

$$
\begin{aligned}
& \Delta\left(\alpha_{n}, \ldots, \alpha_{1}\right) \\
& =1_{A^{\prime}} \otimes\left(\alpha_{n}, \ldots, \alpha_{1}\right)+\left(\alpha_{n}, \ldots, \alpha_{1}\right) \otimes 1_{A}+\sum_{l=1}^{n-1}\left(\alpha_{n}, \ldots, \alpha_{l+1}\right) \otimes\left(\alpha_{l}, \ldots, \alpha_{1}\right) .
\end{aligned}
$$

With these definitions $B_{\infty}(\mathcal{A})$ is an augmented DG cocategory.

Remark 3.9. Let $\mathcal{A}$ be an ordered and finite $A_{\infty}$ category such that $\overline{\mathcal{A}}(A, A)=0$ for any object $A \in \mathrm{ob} \mathcal{A}$. Then the $\mathrm{DG}$ cocategory $B_{\infty}(\mathcal{A})$ also satisfies these conditions; i.e. is ordered, finite and $\overline{B_{\infty}(\mathcal{A})}(A, A)=0$ for any object $A$.

Analogously, to an augmented DG cocategory $\mathcal{B}$ via a cobar construction one can assign a DG category $\Omega(\mathcal{B})$. Let $\mathcal{B}$ be a DG cocategory with a differential $d$ and cocomposition $\Delta$. Its cobar DG category is equal to $T\left(S^{-1} \overline{\mathcal{B}}\right)$. Here $S^{-1} \overline{\mathcal{B}}$ denotes the shift of the cocategory $\mathcal{B}$ and $T\left(S^{-1} \overline{\mathcal{B}}\right)$ is the tensor DG category of it. As before, the spaces of morphisms in $T\left(S^{-1} \overline{\mathcal{B}}\right)$ are given by

$$
\begin{aligned}
& \Omega(\mathcal{B})\left(B, B^{\prime}\right)= S^{-1} \overline{\mathcal{B}}\left(B, B^{\prime}\right) \oplus \bigoplus_{B_{1} \in \mathrm{ob}(\mathcal{B})} S^{-1} \overline{\mathcal{B}}\left(B_{1}, B^{\prime}\right) \otimes S^{-1} \overline{\mathcal{B}}\left(B, B_{1}\right) \\
& \oplus \bigoplus_{B_{1}, B_{2} \in \mathrm{ob}(\mathcal{B})} S^{-1} \overline{\mathcal{B}}\left(B_{2}, B^{\prime}\right) \otimes S^{-1} \overline{\mathcal{B}}\left(B_{1}, B_{2}\right) \otimes S^{-1} \overline{\mathcal{B}}\left(B, B_{1}\right) \oplus \ldots
\end{aligned}
$$

for $B \neq B^{\prime}$ and again one has to add $1_{B}$ in degree 0 for $\Omega(\mathcal{B})(B, B)$. The composition in $\Omega(\mathcal{B})$ is defined by concatenation and the differential $\partial$ on the spaces of morphisms is

$$
\partial=\sum 1 \otimes \ldots \otimes 1 \otimes(d+\Delta) \otimes 1 \otimes \ldots \otimes 1 .
$$

Remark 3.10. If an ordered and finite DG cocategory $\mathcal{B}$ satisfies the condition $\overline{\mathcal{B}}(B, B)=0$, then the category $\Omega(\mathcal{B})$ is also finite, ordered and $\overline{\Omega(B)}(B, B)=0$.

For an augmented $A_{\infty}$ category $\mathcal{A}$ its universal DG category $U(A)$ is defined as $\Omega\left(B_{\infty}(\mathcal{A})\right)$. There is a natural map $\mathcal{A} \rightarrow U(\mathcal{A})$. It is proved in LH03 that this map extends to a functor and is an $A_{\infty}$-quasi-equivalence. Moreover, for an $A_{\infty}$-quasi-equivalence $\phi$ the functor $U(\phi)$ is a quasi-equivalence of DG categories.

3.1.4. $A_{\infty}$ modules.

Definition 3.11. An $A_{\infty}$ module over an $A_{\infty}$ category $\mathcal{A}$ is an $A_{\infty}$ functor $M: \mathcal{A} \rightarrow$ DGVect $_{\mathbb{C}}$. A morphism of modules $G: M \rightarrow N$ is given by a family 
$\left\{G_{A}: M_{0}(A) \rightarrow N_{0}(A)\right\}_{A \in \mathrm{ob} \mathcal{A}}$ of morphisms in DGVect $\mathbb{C}$ such that the diagrams

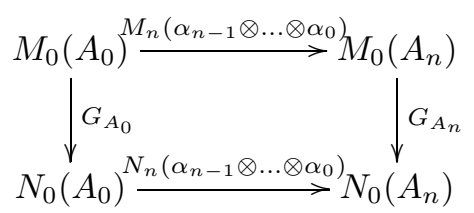

commute for any $n \in \mathbb{N}, A_{i} \in$ ob $\mathcal{A}$ and $\alpha_{i} \in \mathcal{A}\left(A_{i}, A_{i+1}\right)$.

Following LH03, the category of $A_{\infty}$ modules over an $A_{\infty}$ category $\mathcal{A}$ will be denoted as $\operatorname{Mod}_{\infty}^{\text {strict }} \mathcal{A}$.

The morphism $G: M \rightarrow N$ of modules is an $A_{\infty}$-quasi-isomorphism if $G_{A}$ is a quasi-isomorphism of complexes for any $A \in \mathrm{ob} \mathcal{A}$.

The derived category $D_{\infty}(\mathcal{A})$ of an $A_{\infty}$ category $\mathcal{A}$ is defined as a localization of the category $\operatorname{Mod}_{\infty}^{\text {strict }} \mathcal{A}$ with respect to the class of $A_{\infty}$-quasi-isomorphisms. For $A_{\infty}$-quasi-equivalent $A_{\infty}$ categories the derived categories are equivalent.

Remark 3.12 (Lemme 2.4.3.2 of [LH03]). For a DG category $\mathcal{C}$ the derived categories $D(\mathcal{C})$ and $D_{\infty}(\mathcal{C})$ are equivalent. Hence, for any augmented $A_{\infty}$ category $\mathcal{A}$ the category $D_{\infty}(\mathcal{A})$ is equivalent to $D(U(\mathcal{A}))$.

Theorem 3.13. Let $\mathcal{C}$ be a $D G$ category with finitely many objects. Assume that $H(\mathcal{C})$ is an ordered and finite graded category such that $H(\mathcal{C})(C, C)=\mathbb{C}$ for any object $C$ of $\mathcal{C}$. Then there exists an ordered and finite $D G$ category $\widetilde{\mathcal{C}}$ such that $D(\mathcal{C})$ is equivalent to $D(\widetilde{\mathcal{C}})$.

Proof. Theorem 3.6 guarantees existence of a minimal model of $\mathcal{C}-$ an $A_{\infty}$ category $H(\mathcal{C})$. By Remark 3.7 we can assume that $H(\mathcal{C})$ is strictly unital. As $H(\mathcal{C})(C, C)=\mathbb{C}$ for any $C$ the category $H(C)$ is an augmented ordered $A_{\infty}$ category. We have

$$
\mathcal{D}=D\left(\mathcal{C}_{\sigma}\right)=D_{\infty}\left(H\left(\mathcal{C}_{\sigma}\right)\right)=D\left(\mathcal{U}\left(H\left(\mathcal{C}_{\sigma}\right)\right)\right) .
$$

Remarks 3.9 and 3.10 show that the category $\mathcal{U}\left(H\left(\mathcal{C}_{\sigma}\right)\right)$ is ordered and finite.

As a corollary we get

Theorem 3.14. Let $X$ be a smooth projective variety and let $\sigma=\left\langle\mathcal{E}_{1}, \ldots, \mathcal{E}_{n}\right\rangle$ be a full exceptional collection on $X$. Then there exists an ordered, finite $D G$ category $\widetilde{\mathcal{C}_{\sigma}}$ such that $D^{b}(X)$ is equivalent to $D^{b}\left(\widetilde{\mathcal{C}_{\sigma}}\right)$.

Proof. By Theorem 2.3 there exists a DG category $\mathcal{C}_{\sigma}$ such that $D^{b}(X)=D^{b}\left(\mathcal{C}_{\sigma}\right)$. As sheaves $\mathcal{E}_{1}, \ldots, \mathcal{E}_{n}$ are exceptional and the category $\operatorname{Coh}(X)$ is Ext-finite, the category $\mathcal{C}_{\sigma}$ satisfies the conditions of Theorem 3.13 and there exists a DG category $\widetilde{\mathcal{C}_{\sigma}}$ such that categories $D\left(\mathcal{C}_{\sigma}\right)$ and $D \widetilde{\left(\mathcal{C}_{\sigma}\right)}$ are equivalent. As $D^{b}\left(\mathcal{C}_{\sigma}\right) \subset D\left(\mathcal{C}_{\sigma}\right)$ is the subcategory of compact objects, it follows that $D^{b}\left(\mathcal{C}_{\sigma}\right)=D^{b}\left(\widetilde{\left.\mathcal{C}_{\sigma}\right)}\right.$.

The category $\widetilde{\mathcal{C}_{\sigma}}$ is $A_{\infty}$-quasi-equivalent to the category $\mathcal{C}_{\sigma}$ and hence $H^{k}\left(\widetilde{\mathcal{C}_{\sigma}}\left(\mathcal{E}_{i}, \mathcal{E}_{j}\right)\right)=\operatorname{Ext}_{X}^{k}\left(\mathcal{E}_{i}, \mathcal{E}_{j}\right)$.

To graphically present the DG category $\widetilde{\mathcal{C}_{\sigma}}$ we introduce the following definition. 
A $D G$ quiver is a quiver $Q$ endowed with a $\mathbb{Z}$-grading on the set of arrows $Q_{1}$ and a structure of a DG algebra on the path algebra $\mathbb{C} Q$ such that the following conditions are satisfied:

- The $\mathbb{Z}$-grading of $\mathbb{C} Q$ is compatible with the grading of $Q_{1}$.

- For any vertex $q \in Q_{0}$ the trivial path $e_{q} \in \mathbb{C} Q$ is closed of degree 0 .

The DG category $\widetilde{\mathcal{C}_{\sigma}}$ has finite-dimensional spaces of morphisms and so one can associate to it a DG quiver $Q_{\sigma}$. If the collection $\sigma$ is strong, the DG quiver coincides with the quiver introduced in [Bon89].

\section{Calculating the DG Category $\widetilde{\mathcal{C}_{\sigma}}$}

We present two algorithms allowing us to calculate the DG category $\widetilde{\mathcal{C}_{\sigma}}$. The first one goes back to BK90] and uses mutations of exceptional collections. The second one is based on universal extensions defined by Hille and Perling in [HP11].

4.1. Action of the braid group. We have seen that the braid group acts on the set of exceptional collections. This action lifts to associated DG categories.

Twisted complexes provide a description of the categories $\widetilde{\mathcal{C}_{L_{i} \sigma}}$ and $\widetilde{\mathcal{C}_{R_{i} \sigma}}$ by means of $\widetilde{\mathcal{C}_{\sigma}}$. To see this we need to define a tensor product of a twisted complex with a complex of vector spaces.

Let $\mathcal{C}$ be a finite $\mathrm{DG}$ category, let $C \in \mathcal{C}^{\text {pre-tr }}$ be a twisted complex and let $V^{\bullet}$ be a finite-dimensional complex of vector spaces with the differential $\partial^{i}: V^{i} \rightarrow V^{i+1} . C \otimes V$ is defined as $\left(\bigoplus_{\left\{i \mid V^{i} \neq 0\right\}} C[-i]^{\oplus \operatorname{dim} V^{i}}, q_{i, j}\right) \in\left(\mathcal{C}^{\text {pre-tr }}\right)^{\text {pre-tr }}$. The morphisms $q_{i, i+1}$ are induced by the differential $\partial^{i}$ tensored with the identity on $C$ and $q_{i, j}=0$ for $j \neq i+1$.

Now, let $C, D \in \mathcal{C}^{\text {pre-tr }}$ be twisted complexes. There exist closed morphisms of degree 0:

$$
\begin{aligned}
& \phi: C \otimes \mathcal{H o m}_{\mathcal{C}^{\text {pre-tr }}}(C, D) \rightarrow D, \\
& \psi: C \rightarrow \mathcal{H o m}_{\mathcal{C}^{\text {pre-tr }}}(C, D)^{*} \otimes D .
\end{aligned}
$$

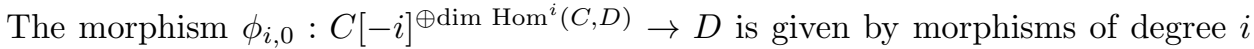
between $C$ and $D ; \psi$ is defined analogously. Now we define new twisted complexes over $\mathcal{C}$ :

$$
L_{C} D=\operatorname{Tot}(\operatorname{Cone}(\phi)[-1]), \quad R_{D} C=\operatorname{Tot}(\operatorname{Cone}(\psi)) .
$$

Let $\sigma=\left\langle\mathcal{E}_{1}, \ldots, \mathcal{E}_{n}\right\rangle$ be a full exceptional collection on a smooth projective variety $X$ and let $\widetilde{\mathcal{C}_{\sigma}}$ be the category described in Theorem 3.13. Let $E_{1}, \ldots, E_{n}$ denote the objects of $\widehat{\mathcal{C}}_{\sigma}$. We define two full subcategories of $\widetilde{\mathcal{C}_{\sigma}}{ }^{\text {pre-tr }}: \widetilde{\mathcal{C}_{\sigma}^{L_{i}}}$ with objects $E_{1}, \ldots, E_{i}, L_{E_{i}} E_{i+1}, E_{i+2}, \ldots, E_{n}$ and $\widetilde{\mathcal{C}_{\sigma}^{R_{i}}}$ with $E_{1}, \ldots, E_{i-1}, R_{E_{i+1}} E_{i}, E_{i+1}, \ldots, E_{n}$.

Proposition 4.1. Let $\sigma=\left\langle\mathcal{E}_{1}, \ldots, \mathcal{E}_{n}\right\rangle$ be a full exceptional collection on $X$ and let $\widetilde{\mathcal{C}_{\sigma}}$ be a finite $D G$ category with objects $E_{1}, \ldots, E_{n}$ such that $H^{k} \mathcal{C}_{\sigma}\left(E_{i}, E_{j}\right)=$ $\operatorname{Ext}_{X}^{k}\left(\mathcal{E}_{i}, \mathcal{E}_{j}\right)$ and $D^{b}(X)$ is equivalent to $D^{b}\left(\widetilde{\mathcal{C}_{\sigma}}\right)$. Then the categories $\widetilde{\mathcal{C}_{\sigma}^{L_{i}}}$ and $\widetilde{\mathcal{C}_{\sigma}^{R_{i}}}$ satisfy analogous conditions for the collections $L_{i} \sigma$ and $R_{i} \sigma$ respectively.

Proof. As the category $\widetilde{\mathcal{C}_{\sigma}}$ is finite, mutations of twisted complexes over $\widetilde{\mathcal{C}_{\sigma}}$ are well defined. Furthermore, the category $D^{b}(X)$ is equivalent to $\widetilde{\mathcal{C}}_{\sigma}^{\text {tr }}$. Under this 
equivalence $L_{\mathcal{E}_{i}} \mathcal{E}_{i+1}$ corresponds to $L_{E_{i}} E_{i+1}$. Hence the category $\widetilde{\mathcal{C}_{\sigma}^{L_{i}}}$ is the DG category described by Theorem 2.3 . By construction it is also finite.

4.2. Universal extensions. Let $E, F$ be objects of a $\mathbb{C}$-linear abelian category. Note that $\operatorname{End}\left(\operatorname{Ext}^{1}(E, F)\right)=\operatorname{Ext}^{1}\left(E, F \otimes \operatorname{Ext}^{1}(E, F)^{*}\right)$. As id $\in \operatorname{End}\left(\operatorname{Ext}^{1}(E, F)\right)$

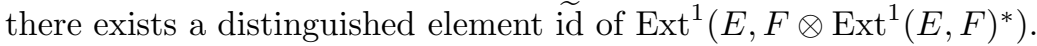

Following [HP11] we say that an object $\bar{E}$ is the universal extension of $E$ by $F$ if $\bar{E}$ is the extension of $E$ by $F \otimes \operatorname{Ext}^{1}(E, F)^{*}$ corresponding to $\widetilde{\text { id }} \in$ $\operatorname{Ext}^{1}\left(E, F \otimes \operatorname{Ext}^{1}(E, F)^{*}\right) . \bar{E}$ fits into the short exact sequence

$$
0 \longrightarrow F \otimes \operatorname{Ext}^{1}(E, F)^{*} \longrightarrow \bar{E} \longrightarrow E \longrightarrow 0 \text {. }
$$

This short exact sequence gives the long exact sequence

$$
0 \longrightarrow \operatorname{Hom}(E, F) \longrightarrow \operatorname{Hom}(\bar{E}, F) \longrightarrow \operatorname{Hom}(F, F) \otimes \operatorname{Ext}^{1}(E, F) \longrightarrow \ldots
$$

This shows that $\operatorname{Ext}^{1}(\bar{E}, F)=0$ and the groups $\operatorname{Ext}^{n}(\bar{E}, F)$ and $\operatorname{Ext}^{n}(E, F)$ are isomorphic for $n>1$ if $\operatorname{Ext}^{n}(F, F)=0$ for $n>0$.

Moreover, if $\operatorname{Ext}^{n}(F, E)=0$ for $n \geq 0$ and $\operatorname{Hom}(F, F)=\mathbb{C}$ and $\operatorname{Ext}^{q}(F, F)=0$ for $q>0$, then the long exact sequence

$$
0 \longrightarrow \operatorname{Hom}(F, F) \otimes \operatorname{Ext}^{1}(E, F)^{*} \longrightarrow \operatorname{Hom}(F, \bar{E}) \longrightarrow \operatorname{Hom}(F, E) \longrightarrow \ldots
$$

shows that $\operatorname{Hom}(F, \bar{E})=\operatorname{Ext}^{1}(E, F)^{*}$.

Thus, if $F$ is exceptional and $\operatorname{Ext}^{n}(F, E)=0$ for all $n$, the objects $\bar{E}, F$ and morphisms between them determine $E$ - as a cone of the canonical morphism

$$
F \otimes \mathcal{H o m}(F, \bar{E}) \stackrel{\text { can }}{\longrightarrow} \bar{E} \longrightarrow E .
$$

Assume that $(E, F)$ is an exceptional pair in an enhanced triangulated category with the enhancement $\widetilde{\mathcal{C}}$ and denote by $\mathcal{C}$ the DG subcategory of $\widetilde{\mathcal{C}}$ with objects $\bar{E}$ and $F$. Then the cone of the canonical morphism $F \otimes \mathcal{H o m}(F, \bar{E}) \rightarrow \bar{F}$ in $\mathcal{C}^{\text {pre-tr }}$ corresponds to $E$. Hence, as in the case of mutations, knowing the DG subcategory of $\widetilde{\mathcal{C}}$ with objects $\bar{E}$ and $F$ we can calculate the DG subcategory with objects $E$ and $F$.

Theorem 4.2. Let $\sigma=\left\langle\mathcal{E}_{1}, \ldots, \mathcal{E}_{n}\right\rangle$ be a full exceptional collection on a smooth projective variety $X$ such that $\operatorname{Ext}^{k}\left(\mathcal{E}_{i}, \mathcal{E}_{j}\right)=0$ for $k \neq 0,1$ and any $i, j$. The $D G$ category $\widetilde{\mathcal{C}_{\sigma}}$ can be calculated by means of universal extensions.

Proof. Following [HP11] we define $\mathcal{E}_{i}(1)=\mathcal{E}_{i}(2)=\ldots=\mathcal{E}_{i}(i)=\mathcal{E}_{i}$ and for $j>i$ we put $\mathcal{E}_{i}(j)$ to be the universal extension of $\mathcal{E}_{i}(j-1)$ by $\mathcal{E}_{j}$ :

$$
0 \longrightarrow \mathcal{E}_{j} \otimes \operatorname{Ext}^{1}\left(\mathcal{E}_{i}(j-1), \mathcal{E}_{j}\right)^{*} \longrightarrow \mathcal{E}_{i}(j) \longrightarrow \mathcal{E}_{i}(j-1) \longrightarrow 0 .
$$

Then $\mathcal{F}=\bigoplus_{i=1}^{n} \mathcal{E}_{i}(n)$ is a tilting object - it generates $D^{b}(X)$ and $\operatorname{Ext}^{i}(\mathcal{F}, \mathcal{F})=$ 0 for $i>0$. Moreover, as the $\mathcal{E}_{i}$ 's are exceptional and there are no morphisms from $\mathcal{E}_{j}$ to $\mathcal{E}_{i}(j-1)$, the objects $\mathcal{E}_{1}(n), \ldots, \mathcal{E}_{n}(n)$ determine the $\mathcal{E}_{i}$ 's. Thus, as described above, the endomorphism algebra of $\bigoplus_{i=1}^{n} \mathcal{E}_{i}(n)$ determines the DG structure of the collection $\left\langle\mathcal{E}_{1}, \ldots, \mathcal{E}_{n}\right\rangle$. 
4.3. Example. In Bod13 we shall describe how universal extensions and mutations allow us to calculate the DG category $\widetilde{\mathcal{C}_{\sigma}}$ for exceptional collections of line bundles on rational surfaces. Here we present the first nontrivial example.

Let $X$ be the blow up of $\mathbb{P}^{2}$ along a subscheme of degree 2 supported at a point $x_{0}$. The Picard group of $X$ is generated by divisors $E_{1}, E_{2}$ and $H$ with intersection form given by

$$
E_{1}^{2}=-2, \quad E_{2}^{2}=-1, \quad H^{2}=1, \quad E_{1} E_{2}=1, \quad E_{i} H=0 .
$$

Let $D_{1}$ denote the strict transform of a line on $\mathbb{P}^{2}$ such that $D_{1} \cap E_{1} \neq \emptyset$ and analogously let $D_{2}$ be a strict transform of a line on $\mathbb{P}^{2}$ such that $D_{2} \cap E_{2} \neq \emptyset$. Finally, let $D_{3}$ be a strict transform of a line on $\mathbb{P}^{2}$ such that $E_{1} \cap D_{3}=\emptyset=E_{2} \cap D_{3}$.

The collection $\left\langle\mathcal{O}_{X}, \mathcal{O}_{X}\left(E_{2}\right), \mathcal{O}_{X}\left(E_{1}+E_{2}\right), \mathcal{O}_{X}(H), \mathcal{O}_{X}(2 H)\right\rangle$ is full and exceptional with an Ext-quiver

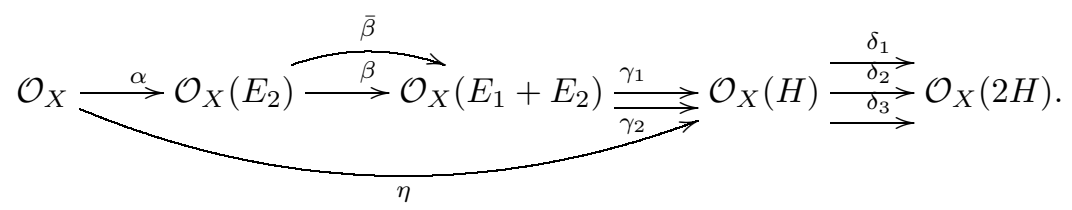

Here, $\bar{\beta}$ denotes the nonzero element of $\operatorname{Ext}^{1}\left(\mathcal{O}_{X}\left(E_{2}\right), \mathcal{O}_{X}\left(E_{1}+E_{2}\right)\right)=\mathbb{C}$.

Then relations between these morphisms are

$$
\begin{array}{ll}
\delta_{1} \gamma_{2}=\delta_{2} \gamma_{1}, & \delta_{1} \eta=\delta_{3} \gamma_{1} \beta \alpha, \\
\delta_{2} \eta=\delta_{3} \gamma_{2} \beta \alpha, & \bar{\beta} \alpha=0, \\
\gamma_{1} \bar{\beta}=0, & \gamma_{2} \bar{\beta}=0 .
\end{array}
$$

In order to calculate the DG quiver of this collection, either we have to mutate it to a strong collection $\left\langle\mathcal{O}_{E_{2}}\left(E_{2}\right)[-1], \mathcal{O}_{X}, \mathcal{O}_{X}\left(E_{1}+E_{2}\right), \mathcal{O}_{X}(H), \mathcal{O}_{X}(2 H)\right\rangle$ or to calculate the endomorphism algebra of an object $\mathcal{O}_{X} \oplus V \oplus \mathcal{O}_{X}\left(E_{1}+E_{2}\right) \oplus \mathcal{O}_{X}(H) \oplus$ $\mathcal{O}_{X}(2 H)$, where $V$ is defined by a nontrivial extension of $\mathcal{O}_{X}\left(E_{2}\right)$ by $\mathcal{O}_{X}\left(E_{1}+E_{2}\right)$ :

$$
0 \longrightarrow \mathcal{O}_{X}\left(E_{1}+E_{2}\right) \stackrel{\phi_{1}}{\longrightarrow} V \stackrel{\phi_{2}}{\longrightarrow} \mathcal{O}_{X}\left(E_{2}\right) \longrightarrow 0 .
$$

In both cases, up to a quasi-isomorphism, we obtain the following DG quiver:

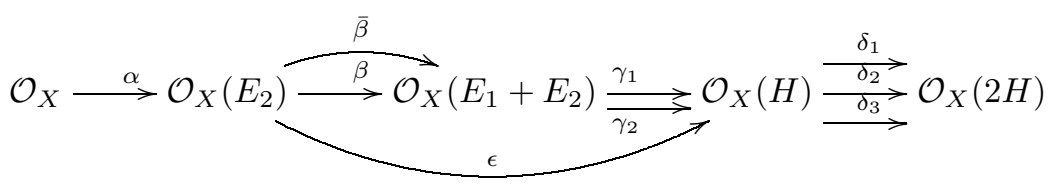

with $\bar{\beta}$ in degree 1 and remaining arrows in degree 0 . Differentials and relations in this DG quiver are

$$
\begin{array}{lll}
\delta_{1} \gamma_{2}=\delta_{2} \gamma_{1}, & \delta_{1} \eta=\delta_{3} \gamma_{1} \beta \alpha, & \delta_{2} \eta=\delta_{3} \gamma_{2} \beta \alpha, \\
\bar{\beta} \alpha=0, & \gamma_{1} \bar{\beta}=0, & \gamma_{2} \bar{\beta}=0 .
\end{array}
$$

4.3.1. Noncommutative deformation. As before, let $X$ be the blow up of $\mathbb{P}^{2}$ along the subscheme of degree 2 supported at a point $x_{0}$ and let $Y$ be the blow up of $\mathbb{P}^{2}$ along two different points $x_{0}$ and $x_{1}$.

The Picard group of $X$ is generated by divisors $E_{1}, E_{2}$ and $H_{X}$ while the Picard group of $Y$ is generated by divisors $F_{1}, F_{2}$ and $H_{Y}$ such that $F_{1}^{2}=-1=F_{2}^{2}$, $F_{1} F_{2}=0, F_{i} H_{Y}=0$ and $H_{Y}^{2}=0$. 
On $X$ the exceptional collection $\sigma=\left\langle\mathcal{O}_{X}, \mathcal{O}_{X}\left(E_{2}\right), \mathcal{O}_{X}\left(E_{1}+E_{2}\right), \mathcal{O}_{X}\left(H_{X}\right)\right.$, $\left.\mathcal{O}_{X}\left(2 H_{X}\right)\right\rangle$ is full and the exceptional collection $\tau=\left\langle\mathcal{O}_{Y}, \mathcal{O}_{Y}\left(F_{2}\right), \mathcal{O}_{Y}\left(F_{1}\right), \mathcal{O}_{Y}\left(H_{Y}\right)\right.$, $\left.\mathcal{O}_{Y}\left(2 H_{Y}\right)\right\rangle$ is full on $Y$.

Let $t \in \mathbb{C}$ be a parameter and consider a DG quiver $Q(t)$ :

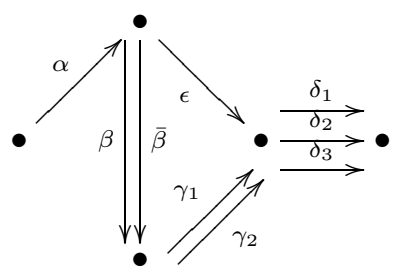

with $\bar{\beta}$ in degree 1 , remaining arrows in degree 0 , differentials

$$
\partial(\beta)=t \bar{\beta}, \quad \partial(\epsilon)=\gamma_{1} \bar{\beta}
$$

and relations

$$
\begin{aligned}
& \gamma_{2} \bar{\beta}=0, \quad \bar{\beta} \alpha=0, \\
& \delta_{1} \gamma_{2}=\delta_{2} \gamma_{1}, \quad \delta_{1} \epsilon=\delta_{3} \gamma_{1} \beta, \quad \delta_{2} \epsilon=\delta_{3} \gamma_{2} \beta \text {, } \\
& \delta_{1} \gamma_{1} \bar{\beta}=0, \quad \delta_{2} \gamma_{1} \bar{\beta}=0 .
\end{aligned}
$$

Let $D(t)$ denote the DG algebra of paths of this DG quiver.

For $t=0, Q(t)$ is the DG quiver of the full collection $\sigma$ on $X$.

For $t \neq 0, D(t)$ has cohomology groups concentrated in degree 0 and hence is quasi-isomorphic to the path algebra of the following quiver:

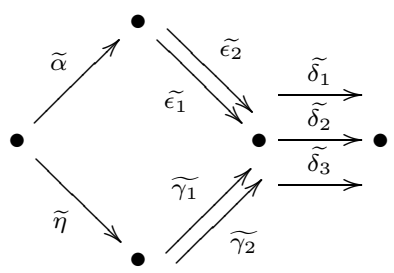

with

$$
\begin{aligned}
& \widetilde{\alpha}=\alpha, \quad \tilde{\eta}=\beta \alpha, \quad \widetilde{\epsilon_{1}}=\gamma_{1} \beta-t \epsilon, \\
& \widetilde{\epsilon_{2}}=\gamma_{2} \beta, \quad \tilde{\gamma_{1}}=\gamma_{1}, \quad \tilde{\gamma_{2}}=\gamma_{2} \text {, } \\
& \widetilde{\delta_{1}}=\delta_{1}, \quad \widetilde{\delta_{2}}=\delta_{2}, \quad \widetilde{\delta_{3}}=\delta_{1}-t \delta_{3} .
\end{aligned}
$$

Relations between morphisms are:

$$
\begin{aligned}
& \widetilde{\epsilon_{2}} \widetilde{\alpha}=\gamma_{2} \beta \alpha=\widetilde{\gamma_{2}} \widetilde{\eta}, \\
& \widetilde{\delta_{1}} \widetilde{\gamma_{2}}=\delta_{1} \gamma_{2}=\delta_{2} \gamma_{1}=\widetilde{\delta_{2}} \widetilde{\gamma_{1}}, \\
& \widetilde{\delta_{2}} \widetilde{\epsilon_{1}}=\delta_{2} \gamma_{1} \beta-t \delta_{2} \epsilon=\delta_{1} \gamma_{2} \beta-t \delta_{3} \gamma_{2} \beta=\widetilde{\delta_{3}} \widetilde{\epsilon_{2}} .
\end{aligned}
$$

Hence, for $t \neq 0$ the quiver $Q(t)$ is quasi-isomorphic to the quiver of the full collection $\tau$ on $Y$, and the path algebra $D(t)$ is an example of a noncommutative deformation of $X$ to $Y$. 


\section{ACKNOWLEDGEMENTS}

The author is greatly indebted to Professor Alexey Bondal for the introduction to the problem and many fruitful discussions, and to Professor Adrian Langer for drawing the author's attention to the paper HP11 and for a lot of help with the computations.

\section{REFERENCES}

[AKO06] Denis Auroux, Ludmil Katzarkov, and Dmitri Orlov, Mirror symmetry for del Pezzo surfaces: vanishing cycles and coherent sheaves, Invent. Math. 166 (2006), no. 3, 537582, DOI 10.1007/s00222-006-0003-4. MR2257391 (2007g:14045)

[BK90] A. I. Bondal and M. M. Kapranov, Framed triangulated categories (Russian), Mat. Sb. 181 (1990), no. 5, 669-683; English transl., Math. USSR-Sb. 70 (1991), no. 1, 93-107. MR:1055981 (91g:18010)

[BLL04] Alexey I. Bondal, Michael Larsen, and Valery A. Lunts, Grothendieck ring of pretriangulated categories, Int. Math. Res. Not. 29 (2004), 1461-1495, DOI 10.1155/S1073792804140385. MR2051435 (2005d:18014)

[Bod13] Agnieszka Bodzenta. Dg categories of smooth rational surfaces. arXiv preprint arXiv:1301.6338 [math.AG], 2013.

[Bon89] A. I. Bondal, Representations of associative algebras and coherent sheaves (Russian), Izv. Akad. Nauk SSSR Ser. Mat. 53 (1989), no. 1, 25-44; English transl., Math. USSRIzv. 34 (1990), no. 1, 23-42. MR992977 (90i:14017)

[HI13] Andreas Hochenegger and Nathan Owen Ilten, Exceptional sequences on rational $\mathbb{C}^{*}$ surfaces, Manuscripta Math. 142 (2013), no. 1-2, 1-34, DOI 10.1007/s00229-012-0591-9. MR3080998

[HP11] Lutz Hille and Markus Perling, Exceptional sequences of invertible sheaves on rational surfaces, Compos. Math. 147 (2011), no. 4, 1230-1280, DOI 10.1112/S0010437X10005208. MR2822868 (2012g:14026)

[Kad82] T. V. Kadeishvili, The algebraic structure in the homology of an $A(\infty)$-algebra (Russian, with English and Georgian summaries), Soobshch. Akad. Nauk Gruzin. SSR 108 (1982), no. 2, 249-252 (1983). MR720689 (84k:55009)

[Kel94] Bernhard Keller, Deriving DG categories, Ann. Sci. École Norm. Sup. (4) 27 (1994), no. 1, 63-102. MR1258406 (95e:18010)

[Kel06] Bernhard Keller, A-infinity algebras, modules and functor categories, Trends in representation theory of algebras and related topics, Contemp. Math., vol. 406, Amer. Math. Soc., Providence, RI, 2006, pp. 67-93, DOI 10.1090/conm/406/07654. MR2258042 (2007g:18002)

[LH03] Kenji Lefevre-Hasegawa, Sur les a-infini catégories, arXiv preprint math/0310337, 2003.

[Or192] D. O. Orlov, Projective bundles, monoidal transformations, and derived categories of coherent sheaves (Russian, with Russian summary), Izv. Ross. Akad. Nauk Ser. Mat. 56 (1992), no. 4, 852-862, DOI 10.1070/IM1993v041n01ABEH002182; English transl., Russian Acad. Sci. Izv. Math. 41 (1993), no. 1, 133-141. MR.1208153(94e:14024)

[Per10] Markus Perling, Examples for exceptional sequences of invertible sheaves on rational surfaces, Geometric Methods in Representation Theory II, Seminaire et Congres(25):369-389, 2010.

Faculty of Mathematics, Informatics and Mechanics, University of Warsaw, Banacha

2, 02-097 WARSAW, POLAND

E-mail address: a.bodzenta@mimuw.edu.pl

Current address: National Research University Higher School of Economics, Department of Mathematics, 20 Myasnitskaya St, Moscow 101000 Russia

E-mail address: abodzenta@hse.ru 